\title{
On exact solution of unsteady MHD flow of a viscous fluid in an orthogonal rheometer
}

\author{
Muhammad Afzal Rana', Sadia Siddiqa ${ }^{2 *}$ and Saima Noor ${ }^{3}$
}

"Correspondence:

saadiasiddiqa@gmail.com

${ }^{2}$ Department of Mathematics, COMSATS Institute of Information

Technology, Attock, Pakistan Full list of author information is

available at the end of the article

\begin{abstract}
This paper studies the unsteady MHD flow of a viscous fluid in which each point of the parallel planes are subject to the non-torsional oscillations in their own planes. The streamlines at any given time are concentric circles. Exact solutions are obtained and the loci $\Gamma$ of the centres of these concentric circles are discussed. It is shown that the motion so obtained gives three infinite sets of exact solutions in the geometry of an orthogonal rheometer in which the above non-torsional oscillations are superposed on the disks. These solutions reduce to a single unique solution when symmetric solutions are looked for. Some interesting special cases are also obtained from these solutions.
\end{abstract}

Keywords: viscous fluid; MHD flow; orthogonal rheometer; eccentric rotation; exact solutions

\section{Introduction}

Berker [1] has defined the 'pseudo plane motions' of the first kind that: if the streamlines in a plane flow are contained in parallel planes but the velocity components are dependent on the coordinate normal to the planes. Berker [2] has obtained a class of exact solutions to the Navier-Stokes equations belonging to the above type of flows. The ingenious analysis of Berker [3, 4] of the flow of an incompressible viscous fluid confined between two coaxially or non-coaxially rotating infinite parallel disks has shown the existence of an infinite number of exact solutions characterizing the asymmetric and symmetric motions. The symmetric solutions presented by Berker [3] belong to a new class and cannot be obtained as limiting cases of any of the known solutions. It was observed that these results are relevant in understanding the motions of the fluid in a device called an orthogonal rheometer.

Owing to the importance of exact solutions, many authors [5-9] have analyzed the flows in this geometry for different types of fluids. Rao and Kasiviswanathan [10] have investigated the unsteady viscous flow in the orthogonal rheometer and established the existence of three infinite sets of exact solutions. The flow of an elastico-viscous fluid in the above geometry has been investigated by Abbott and Walters [11]. They obtained the unique exact solution of the problem and it was considered as a particular case of the infinite set of solutions which satisfies the condition for symmetric solutions. Thornley [12] has considered an exact solution for the flow of an incompressible fluid confined between two infinite disks when both the fluid and the disk were in rigid-body rotation with constant

(c) 2014 Rana et al.; licensee Springer. This is an Open Access article distributed under the terms of the Creative Commons Attribution License (http://creativecommons.org/licenses/by/2.0), which permits unrestricted use, distribution, and reproduction in any medium, provided the original work is properly credited. 
angular velocity and one of the disks is used to perform non-torsional oscillations in its own plane in a rotating frame of reference.

The MHD flow of Newtonian and non-Newtonian fluids between two non-coaxial rotating disks has been one of the subjects which has attracted attention of many workers. Kasiviswanathan and Rao [13] investigated the unsteady MHD flow of a viscous, homogeneous, incompressible, electrically conducting fluid between two infinite, parallel, insulated disks rotating with the same angular velocity about two non-coincident axes in which the disks were subjected to non-torsional oscillations. Using the Laplace transform two sets of infinite solutions are presented and these solutions reduce to unique solution when symmetric solutions are looked for. Mohanty [14] obtained an exact solution for the viscous, homogeneous, incompressible, conducting fluid between eccentrically rotating disks having the same angular velocity in the presence of uniform transverse magnetic field. Further, Erkman [15] studied the same problem but for an induced magnetic field. Murthy and Ram [16] examined an exact solution for the case of flow due to noncoaxial rotations of a porous disk and a fluid at infinity under the application of a uniform transverse magnetic field. Rao and Rao [17] also considered the same physical situation as discussed by [14] but they considered the second grade fluid. Ersoy [18] obtained an exact solution of an Oldroyd-B fluid between two infinite, parallel, insulated disks rotating about non-coincident axes in the presence of a uniform transverse magnetic field. Moreover, Rajagopal [19] reviewed problems related to both Newtonian and non-Newtonian fluids between two parallel disks rotating about the same or distinct axes with the same and different angular velocities.

In this paper we have generalised the work of Rao and Kasiviswanathan [10] to the case of unsteady MHD flow of a conducting, incompressible viscous fluid between two infinite, parallel, insulated disks rotating with the same angular velocity about non-coincident axes normal to the disks. Each point of the parallel planes is subjected to non-torsional oscillations in their own planes. The streamlines at a given instant are concentric circles in each plane parallel to a fixed plane $\pi_{0}$ and each point of the plane is performing non-torsional oscillations. Exact solutions are obtained and the loci of the centres of concentric circles are discussed. In the geometry of an orthogonal rheometer where non-torsional oscillations are imposed on the disks relative to the basic rotations, the existence of three infinite sets of exact solutions is established. Three cases arise i.e. when: (i) $\omega>\delta$, (ii) $\omega<\delta$ and (iii) $\omega=\delta$. These solutions reduce to a single unique solution when symmetric solutions are looked for.

\section{Governing equations}

Consider the flow of an incompressible viscous fluid filling the entire three-dimensional space in which the streamlines at a given instant are concentric circles in each plane parallel to a fixed plane $\pi_{0}$ and each point of the plane is performing non-torsional oscillations. A uniform magnetic field $B_{0}$ is applied along the $z$-axis. The unsteady flow of an incompressible fluid in the presence of a magnetic field is governed by the following equations:

$$
\begin{aligned}
& \rho \frac{D \mathbf{V}}{D t}=\operatorname{div} \mathbf{T}+\mathbf{J} \times \mathbf{B}, \\
& \nabla \cdot \mathbf{V}=0,
\end{aligned}
$$


where $\mathbf{V}$ is the velocity field, $\mathbf{T}$ the Cauchy stress tensor, $\mathbf{J}$ the electric current density, $\rho$ the density and $D / D t$ the material time derivative. In addition, $\mathbf{B}\left(=\mathbf{B}_{0}+\mathbf{b}\right)$ is the total magnetic field in which $\mathbf{b}$ and $\mathbf{B}_{0}$ denote the induced magnetic field and imposed magnetic field, respectively. In the absence of displacement currents, the Maxwell equations [20, 21] and the modified Ohm law [22] can be written as

$$
\left.\begin{array}{l}
\nabla \cdot \mathbf{B}=0, \quad \nabla \times \mathbf{B}=\mu_{m} \mathbf{J}, \quad \nabla \times \mathbf{E}=-\frac{\partial \mathbf{B}}{\partial t}, \\
\mathbf{J}=\sigma[\mathbf{E}+\mathbf{V} \times \mathbf{B}],
\end{array}\right\}
$$

in which $\mathbf{E}$ is the electric field, $\mu_{m}$ the magnetic permeability and $\sigma$ the electric field conductivity. It is assumed that all the thermo-physical fluid properties are constant throughout the flow field region. Also, the induced magnetic field $\mathbf{b}$ is negligible compared with the applied magnetic field $\mathbf{B}_{0}$. It is further supposed that no energy is added or extracted from the fluid by the electric field which implies that there is no electric field present in the fluid flow region. Under these assumptions the electromagnetic force $\mathbf{J} \times \mathbf{B}$ takes the form

$$
\mathbf{J} \times \mathbf{B}=-\sigma B_{0}^{2} \mathbf{V}
$$

The velocity field is chosen as follows:

$$
u=u(x, y, z, t), \quad v=v(x, y, z, t), \quad w=0,
$$

where $u, v, w$ are the components of velocity in the Cartesian coordinate system $(x, y, z)$. For this motion, the streamlines for all time $t$ are contained in planes parallel to the fixed plane $\pi_{0}$ and the plane $O x y$ is parallel to this fixed plane. The constitutive equation of the viscous fluid is

$$
\mathbf{T}=-p \mathbf{I}+\mu \mathbf{A}_{1}+\rho \mathbf{f},
$$

with $p$ being the pressure, $\mathbf{I}$ the unit tensor, $\mathbf{A}_{1}$ the Rivilin-Ericksen tensor, $\mu$ the dynamic viscosity and $\mathbf{f}$ the body force. The Rivilin-Ericksen tensor $\mathbf{A}_{1}$ is defined by

$$
\mathbf{A}_{1}=\mathbf{L}+\mathbf{L}^{T}, \quad \mathbf{L}=\nabla \mathbf{V},
$$

in which $T$ denotes the transpose. The Navier-Stokes equations governing the flow in view of Eqs. (1) and (4)-(7) are given by

$$
\begin{aligned}
& \psi_{y t}+\psi_{y} \psi_{y x}-\psi_{x} \psi_{y y}=-\phi_{x}+v\left(\psi_{x x}+\psi_{y y}+\psi_{z z}\right)_{y}-\frac{\sigma B_{0}^{2} \psi_{y}}{\rho}, \\
& \psi_{x t}+\psi_{y} \psi_{x x}-\psi_{x} \psi_{y x}=\phi_{y}+v\left(\psi_{x x}+\psi_{y y}+\psi_{z z}\right)_{x}-\frac{\sigma B_{0}^{2} \psi_{x}}{\rho}, \\
& u=\psi_{y}, \quad v=-\psi_{x}, \\
& \phi=\frac{p}{\rho}+\Omega
\end{aligned}
$$


where $\psi(x, y, z, t)$ is the stream function, $\Omega$ the potential due to external force and $v$ the kinematic coefficient of viscosity. The subscript denotes the partial derivatives with respect to corresponding variables throughout the paper. From the equation of motion in the $z$-direction one finds $\phi$ to be independent of $z$. In the present analysis the authors are interested in getting solutions of an unsteady MHD fluid motion in which the streamlines at any instant are concentric circles in each plane with normal line parallel to the $\mathrm{Oz}$-axis. Therefore, consider

$$
x=f(z, t), \quad y=g(z, t),
$$

and let these be the loci $\Gamma$ of the centres of these circles which are fixed points at a given instant $t$ and belong to the instantaneous axis of the vortex. The condition that $\Gamma$ must not be a straight line perpendicular to the $O x y$ plane gives the inequality:

$$
f_{z}^{2}+g_{z}^{2} \not 0
$$

Following an analysis similar to Berker [4], the general form of the stream function for this type of unsteady MHD flow is given by

$$
\psi=K\left[(x-f(z, t))^{2}+(y-g(z, t))^{2}\right] .
$$

This stream function $\psi$ has to satisfy Eqs. (8) and (9). According to Eq. (10), the velocity components are given by the equations:

$$
u=2 K(y-g(z, t)), \quad v=-2 K(x-f(z, t)) .
$$

Let $c$ be any constant and let $\pi$ be the plane $z=c$. Equation (15) shows that at a given time $t_{0}$, the fluid particles belonging to the plane $\pi$ move like the points of a solid plate which rotates around a fixed point with coordinates $\left(f\left(c, t_{0}\right), g\left(c, t_{0}\right), c\right)$ and with constant angular velocity $\omega$, given by

$$
\omega=-2 K .
$$

This angular velocity is same for all planes $\pi$ which are parallel to the plane $O x y$. Thus (15) should be interpreted as a motion due to rigid rotation superposed by some unsteady motion.

\section{Exact solutions}

Eliminating $\phi$ from (8) and (9) by differentiating with respect to $z$ and making use of (14) and (16) we obtain

$$
\nu W_{z z z}-\left(\frac{\sigma B_{0}^{2}}{\rho}+i \omega\right) W_{z}=W_{z t},
$$

where

$$
W(z, t)=f(z, t)+i g(z, t) .
$$


We investigate the unsteady MHD motion generated by subjecting each of the parallel planes to a non-torsional oscillation of the type $e^{i \delta t}$ or $e^{-i \delta t}$, where $\delta$ is the frequency of oscillation. Letting

$$
W=F(z) e^{-i \delta t},
$$

Eq. (17) takes the form

$$
F_{z z z}-\left(N+i \frac{\omega-\delta}{v}\right) F_{z}=0
$$

where

$$
N=\frac{\sigma B_{0}^{2}}{\rho v}
$$

The general solutions of the differential equation (20) are

$$
\left.\begin{array}{ll}
F(z)=A+B \exp \left(\sqrt{N+2 i \beta_{1}^{2}} z\right)+C \exp \left(-\sqrt{N+2 i \beta_{1}^{2}} z\right), & \omega>\delta, \\
F(z)=A+B \exp \left(\sqrt{N-2 i \beta_{2}^{2}} z\right)+C \exp \left(-\sqrt{N-2 i \beta_{2}^{2}} z\right), & \omega<\delta, \\
F(z)=A+B \exp (\sqrt{N} z)+C \exp (-\sqrt{N} z), \quad \omega=\delta, &
\end{array}\right\}
$$

where

$$
\left.\begin{array}{l}
\beta_{1}^{2}=(\omega-\delta) / 2 v, \\
\beta_{2}^{2}=(\delta-\omega) / 2 v,
\end{array}\right\}
$$

and the arbitrary complex constants $A, B$ and $C$ are assumed to be

$$
A=A_{1}+i A_{2}, \quad B=B_{1}+i B_{2}, \quad C=C_{1}+i C_{2} .
$$

The solutions for non-torsional oscillation of the type $e^{i \delta t}$ are obtained by replacing $\delta$ by $-\delta$ at appropriate places in the above equations. The general solutions of the differential equation (17) in view of Eqs. (18), (19), (22) and (24) are given by

$$
\begin{aligned}
f= & f_{0}+\left(A_{1} \cos \delta t+A_{2} \sin \delta t\right)+e^{\beta_{r 1} z}\left[B_{1} \cos \left(\beta_{i 1} z-\delta t\right)-B_{2} \sin \left(\beta_{i 1} z-\delta t\right)\right] \\
& +e^{-\beta_{r 1} z}\left[C_{1} \cos \left(\beta_{i 1} z+\delta t\right)+C_{2} \sin \left(\beta_{i 1} z+\delta t\right)\right], \\
g= & g_{0}+\left(A_{2} \cos \delta t-A_{1} \sin \delta t\right)+e^{\beta_{r 1} z}\left[B_{1} \sin \left(\beta_{i 1} z-\delta t\right)+B_{2} \cos \left(\beta_{i 1} z-\delta t\right)\right] \\
& +e^{-\beta_{r 1} z}\left[C_{2} \cos \left(\beta_{i 1} z+\delta t\right)-C_{1} \sin \left(\beta_{i 1} z+\delta t\right)\right],
\end{aligned}
$$

where $f_{0}$ and $g_{0}$ denote solutions due to steady rotation and

$$
\beta_{r 1}+i \beta_{i 1}=\sqrt{N+2 i \beta_{1}^{2}} .
$$

Similarly, expressions for $\omega<\delta$ and $\omega=\delta$ can be obtained. It is worthy to mention that the solutions (25) and (26) reduce to the solutions presented by Rao and Kasiviswanathan [10] when $B_{0}=0$.

Now we will establish that by making a suitable change of axes, that is, the change that satisfies the condition that the plane $O x y$ be parallel to the plane $\pi_{0}$, it is possible to reduce 
the number of arbitrary constants contained in the curve $\Gamma$ given in Eqs. (25) and (26). The change to be made is the result of transformations described below for $\omega>\delta$.

(i) A translation parallel to the plane $O x y$ and defined by the equations:

$$
x_{1}=x-\left(A_{1} \cos \delta t+A_{2} \sin \delta t\right), \quad y_{1}=y-\left(A_{2} \cos \delta t-A_{1} \sin \delta t\right) .
$$

This transformation gets rid of the constants $A_{1}$ and $A_{2}$ contained in Eqs. (25) and (26).

(ii) If $B_{1}^{2}+B_{2}^{2}>0$ and $C_{1}^{2}+C_{2}^{2}>0$, a translation parallel to the axis $O z$ and a rotation about this axis. This translation and rotation is so chosen that the equation of the curve $\Gamma$ in the new axes reduces to the following form:

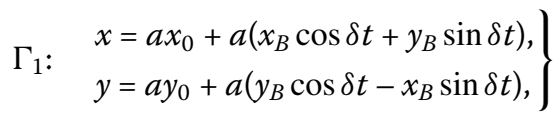

where

$$
\begin{array}{ll}
x_{0}=\cosh \left(\beta_{0} z\right) \cos \left(\beta_{0} z+n\right), & y_{0}=\sinh \left(\beta_{0} z\right) \sin \left(\beta_{0} z+n\right), \\
x_{B}=\cosh \left(\beta_{i 1} z\right) \cos \left(\beta_{i 1} z+n\right), & y_{B}=\sinh \left(\beta_{i 1} z\right) \sin \left(\beta_{i 1} z+n\right)
\end{array}
$$

with $\beta_{1}, a>0, \beta_{0}=\sqrt{\omega / 2 v}>0$. Equation (29) contains only two arbitrary real constants $a$ and $n$.

(iii) If $B_{1}^{2}+B_{2}^{2}>0$ and $C_{1}=C_{2}=0$, a translation parallel to the axis $O z$. This translation is so chosen that the equations of the curve $\Gamma$ with respect to the new axes reduce to the following form:

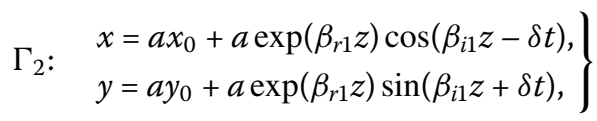

where

$$
x_{0}=\exp \left(\beta_{0} z\right) \cos \left(\beta_{0} z\right), \quad y_{0}=\exp \left(\beta_{0} z\right) \sin \left(\beta_{0} z\right),
$$

with $a>0, \beta_{0} \neq 0, \beta_{1} \neq 0$. Equation (31) involves only one arbitrary real constant $a$.

(iv) If $C_{1}^{2}+C_{2}^{2}>0$ and $B_{1}=0=B_{2}$, again a translation parallel to the axis $O z$. This translation is so chosen that the equations of the curve $\Gamma$ in the new axes reduce to the following form:

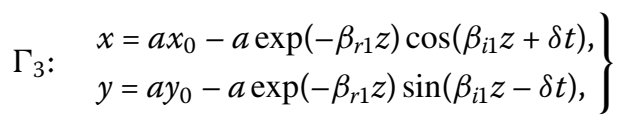

where

$$
x_{0}=\exp \left(-\beta_{0} z\right) \cos \left(\beta_{0} z\right), \quad y_{0}=-\exp \left(-\beta_{0} z\right) \sin \left(\beta_{0} z\right),
$$

with $a>0, \beta_{0} \neq 0, \beta_{1} \neq 0$. Equation (33) again contains only one constant $a$. It is worth mentioning that the curves $\Gamma_{1}-\Gamma_{3}$ reduce to the curves given by Rao and Kasiviswanathan [10] when $B_{0}=0$. 
(v) Lastly, when $\omega=\delta$ the curve $\Gamma$ by a proper choice of axes can be written as

$$
\left.\Gamma_{4}: \begin{array}{l}
x=\left(a z+b z^{2}\right) \cos (\omega t+n), \\
y=-\left(a z+b z^{2}\right) \sin (\omega t+n),
\end{array}\right\}
$$

with $a, b>0 ; n$ is any arbitrary constant. This transformation has no counterpart in the corresponding steady problem. Likewise, curves $\Gamma_{5}$ and $\Gamma_{6}$, similar to the curves $\Gamma_{1}$ and $\Gamma_{2}$, can be obtained for $\omega<\delta$.

Equations (29) of the curve $\Gamma_{1}$ in cylindrical coordinates can be written in the form

$$
r=\frac{a}{2} e^{\left(\beta_{0} z\right)}\left[1+f_{1}\left(\beta_{0}, \beta_{1}, z, t\right)\right]^{1 / 2},
$$

where $f_{1}$ is negligible for large $z$ or for very small $\delta$. So for large $z$ (36) reduces to

$$
r=\frac{a}{2} e^{\beta_{0} z}
$$

Let $C_{1}$ be the projection of the curve $\Gamma_{1}$ on the $O x y$ plane. The equation of $C_{1}$ in polar coordinates is

$$
r=\frac{a}{2} e^{\theta}, \quad \theta=\beta_{0} z
$$

Therefore, the curve $C_{1}$ tends towards the logarithmic spiral (38) for large value of $z$, having the properties given by Berker [4]. A similar discussion holds for the curves $\Gamma_{2}-\Gamma_{6}$. It can be further noted that $e^{\beta_{0} z}$ dominates over the other terms, the curve $\Gamma_{1}$ is wrapped around the surface of revolution $S_{1}$ the axis of which is $\mathrm{Oz}$ and the meridian (in cylindrical coordinates) of which is given in Eq. (36).

\section{Unsteady MHD flow in an orthogonal rheometer}

Consider the unsteady MHD flow of a conducting, incompressible viscous fluid between two infinite, parallel, insulated disks $D_{1}$ and $D_{2}$ rotating with the same angular velocity $\omega$ about non-coincident axes $z^{\prime}$ and $z^{\prime \prime}$ normal to the planes of the disks and performing non-torsional oscillations in their own planes. This model represents the motion of the fluid in the instrument called an orthogonal rheometer.

We choose the Cartesian coordinate system with the $z$-axis perpendicular to the disks and the lower and the upper disks are located at $z=-h$ and $z=h$, respectively. Let the lower disk rotate about the point $P_{1}\left(-x_{1},-y_{1},-h\right)$ and the upper disk about $P_{2}\left(x_{1}, y_{1}, h\right)$. Taking the middle point $O$ of $P_{1} P_{2}$ as the origin and perpendicular axes $O x$ and $O y$ lying in the plane $z=0$ so that the $z$-axis is perpendicular to these axes. The superposed unsteady motion is created by subjecting each point of the lower and upper disk to non-torsional oscillations of the type $\bar{a} e^{i \delta t}+\bar{b} e^{-i \delta t}$ and $\bar{c} e^{i \delta t}+\bar{d} e^{-i \delta t}$, respectively, where $\bar{a}, \bar{b}, \bar{c}$ and $\bar{d}$, are given complex constants. The governing equation for this motion is given by Eq. (17) whereas the boundary conditions are

$$
\begin{aligned}
& W=-\left(x_{1}+i y_{1}\right)+\bar{a} e^{i \delta t}+\bar{b} e^{-i \delta t}, \quad \text { on } z=-h, \\
& W=\left(x_{1}+i y_{1}\right)+\bar{c} e^{i \delta t}+\bar{d} e^{-i \delta t}, \quad \text { on } z=h .
\end{aligned}
$$


Assume the solution for Eq. (17) to be of the form

$$
W=\bar{a} F_{1}(z) e^{i \delta t}+\bar{b} F_{2}(z) e^{-i \delta t}+F_{3}(z)
$$

where the first two terms correspond to the oscillatory motion and the third term gives the solution due to the basic rigid rotation. The corresponding boundary conditions are

$$
F_{1}(-h)=1, \quad F_{2}(-h)=1, \quad F_{3}(-h)=-\left(x_{1}+i y_{1}\right)
$$

and

$$
F_{1}(h)=\frac{\bar{c}}{\bar{a}}, \quad F_{2}(h)=\frac{\bar{d}}{\bar{b}}, \quad F_{3}(h)=x_{1}+i y_{1} .
$$

The governing differential equation (17) is of the third order, giving three sets of infinite solutions; one corresponding to the steady part and the other two corresponding to the oscillatory parts, proportional to $e^{i \delta t}$ and $e^{-i \delta t}$. As there are only two boundary conditions (42) and (43) to determine each complex function, therefore its solution is not unique. We introduce one more arbitrary boundary condition in addition to the boundary conditions (42) and (43) in order to determine the solution. Let $x_{p}$ and $y_{p}$ be the coordinates of the point of intersection of the curve $\Gamma$ and the plane $O x y$ and also the non-torsional oscillations are prescribed on the plane $O x y$. Then the boundary conditions will be of the form

$$
W(0)=x_{P}+i y_{P}+a_{0} e^{i \delta t}+b_{0} e^{-i \delta t}
$$

giving

$$
F_{1}(0)=\frac{a_{0}}{\bar{a}}, \quad F_{2}(0)=\frac{b_{0}}{\bar{b}}, \quad F_{3}(0)=x_{p}+i y_{p} .
$$

The solutions of the differential equation (17), satisfying the boundary conditions (42)(44), are given by

$$
\begin{aligned}
W= & \left\{\frac{\bar{c}+\bar{a}-2 a_{0}}{2} \frac{\cosh \sqrt{N+\lambda_{3}^{2}} z-1}{\cosh \sqrt{N+\lambda_{3}^{2}} h-1}+\frac{\bar{c}-\bar{a}}{2} \frac{\sinh \sqrt{N+\lambda_{3}^{2}} z}{\sinh \sqrt{N+\lambda_{3}^{2}} h}+a_{0}\right\} e^{i \delta t} \\
& +\left\{\frac{\bar{d}+\bar{b}-2 b_{0}}{2} \frac{\cosh \sqrt{N+\lambda_{1}^{2}} z-1}{\cosh \sqrt{N+\lambda_{1}^{2}} h-1}+\frac{\bar{d}-\bar{b} \sinh \sqrt{N+\lambda_{1}^{2}} z}{2} \frac{\sinh \sqrt{N+\lambda_{1}^{2}} h}{\sin } b_{0}\right\} e^{-i \delta t} \\
& +\left(x_{p}+i y_{p}\right)\left\{1-\frac{\cosh \sqrt{N+\lambda_{0}^{2}} z-1}{\cosh \sqrt{N+\lambda_{0}^{2}} h-1}\right\}+\left(x_{1}+i y_{1}\right) \frac{\sinh \sqrt{N+\lambda_{0}^{2}} z}{\sinh \sqrt{N+\lambda_{0}} h},
\end{aligned}
$$

where

$$
\begin{aligned}
& \lambda_{0}^{2}=2 i \beta_{0}^{2}, \quad \lambda_{1}^{2}=2 i \beta_{1}^{2}=-2 i \beta_{2}^{2}, \quad \lambda_{3}^{2}=2 i \beta_{3}^{2}, \quad \beta_{0}^{2}=\frac{\omega}{2 \nu}, \\
& \beta_{1}^{2}=(\omega-\delta) / 2 \nu, \quad \beta_{2}^{2}=(\delta-\omega) / 2 \nu, \quad \beta_{3}^{2}=(\omega+\delta) / 2 \nu .
\end{aligned}
$$


Setting

$$
\begin{array}{lll}
\frac{\bar{c}-\bar{a}}{2}=a_{1}+i a_{2}, & \frac{\bar{c}+\bar{a}-2 a_{0}}{2}=b_{1}+i b_{2}, & a_{0}=a_{1}^{\prime}+i a_{2}^{\prime}, \\
\frac{\bar{d}-\bar{b}}{2}=c_{1}+i c_{2}, & \frac{\bar{d}+\bar{b}-2 b_{0}}{2}=d_{1}+i d_{2}, & b_{0}=b_{1}^{\prime}+i b_{2}^{\prime},
\end{array}
$$

in Eq. (45) and equating the real and imaginary parts, we get for $\omega>\delta$

$$
\begin{aligned}
f= & {\left[\left(c_{2} R_{1}+c_{1} Q_{1}+d_{1} T_{1}+d_{2} S_{1}\right)-\left(a_{2} R_{3}+a_{1} Q_{3}+b_{2} S_{3}+b_{1} T_{3}\right)-a_{2}^{\prime}+b_{2}^{\prime}\right] \sin \delta t } \\
& +\left[\left(a_{1} R_{3}-a_{2} Q_{3}+b_{1} S_{3}-b_{2} T_{3}\right)+\left(c_{1} R_{1}-c_{2} Q_{1}+d_{1} S_{1}-d_{2} T_{1}\right)+a_{1}^{\prime}+b_{1}^{\prime}\right] \cos \delta t \\
& +x_{1} R_{0}-y_{1} Q_{0}+x_{p}\left(1-S_{0}\right)+y_{p} T_{0}
\end{aligned}
$$

and

$$
\begin{aligned}
g= & {\left[\left(a_{1} R_{3}-a_{2} Q_{3}+b_{1} S_{3}-b_{2} T_{3}\right)-\left(c_{1} R_{1}-c_{2} Q_{1}+d_{1} S_{1}-d_{2} T_{1}\right)+a_{1}^{\prime}-b_{1}^{\prime}\right] \sin \delta t } \\
& +\left[\left(a_{2} R_{3}+a_{1} Q_{3}+b_{2} S_{3}+b_{1} T_{3}\right)+\left(c_{2} R_{1}+c_{1} Q_{1}+d_{1} T_{1}+d_{2} S_{1}\right)+a_{2}^{\prime}+b_{2}^{\prime}\right] \cos \delta t \\
& +x_{1} Q_{0}+y_{1} R_{0}-x_{p} T_{0}+y_{p}\left(1-S_{0}\right),
\end{aligned}
$$

where

$$
\frac{\sinh \sqrt{N+\lambda_{j}^{2}} z}{\sinh \sqrt{N+\lambda_{j}^{2}} h}=R_{j} \pm i Q_{j}, \quad \frac{\cosh \sqrt{N+\lambda_{j}^{2}} z-1}{\cosh \sqrt{N+\lambda_{j}^{2}} h-1}=S_{j} \pm i T_{j}, \quad j=0,1,3 .
$$

The minus sign is taken when $j=2$. Thus we have

$$
\begin{aligned}
& R_{j}=\left[\xi_{j}(z) \xi_{j}(h)+\eta_{j}(z) \eta_{j}(h)\right] / \Delta_{1 j}, \quad Q_{j}=\left[\eta_{j}(z) \xi_{j}(h)-\xi_{j}(z) \eta_{j}(h)\right] / \Delta_{1 j}, \\
& S_{j}=\left[\phi_{j}(z) \phi_{j}(h)+\chi_{j}(z) \chi_{j}(h)\right] / \Delta_{2 j}, \quad T_{j}=\left[\chi_{j}(z) \phi_{j}(h)-\phi_{j}(z) \chi_{j}(h)\right] / \Delta_{2 j}, \\
& \xi_{j}(z)=\sinh \left(\beta_{r j} z\right) \cos \left(\beta_{l j} z\right), \quad \eta_{j}(z)=\cosh \left(\beta_{r j} z\right) \sin \left(\beta_{l j} z\right), \\
& \phi_{j}(z)=\cosh \left(\beta_{r j} z\right) \cos \beta_{i j} z-1, \quad \chi_{j}(z)=\sinh \left(\beta_{r j} z\right) \sin \left(\beta_{i j} z\right), \\
& \Delta_{1 j}=\xi_{j}^{2}(h)+\eta_{j}^{2}(h), \quad \Delta_{2 j}=\phi_{j}^{2}(h)+\chi_{j}^{2}(h),
\end{aligned}
$$

where $j=0,1,2,3$. Similarly, we can get the solutions for $\omega<\delta$. The solution for the case $\omega=\delta$ is obtained from Eqs. (48) and (49) by taking the limit $\delta \longrightarrow \omega$. In this case we obtain

$$
R_{1}=R_{2}=\frac{\sinh \sqrt{N} z}{\sinh \sqrt{N} h}, \quad S_{1}=S_{2}=\frac{\cosh \sqrt{N} z-1}{\cosh \sqrt{N} h-1}, \quad Q_{1}=Q_{2}=T_{1}=T_{2}=0 .
$$

We have thus obtained the unsteady MHD motion of viscous fluid in the orthogonal rheometer for the three different cases of $\omega$, i.e., (i) $\omega>\delta$, (ii) $\omega<\delta$, (iii) $\omega=\delta$. The solutions for $f$ and $g$ contain six arbitrary constants, namely $a_{1}, a_{2}, b_{1}, b_{2}, x_{p}$ and $y_{p}$, giving three sets of infinite solutions. Hence the existence of three sets of infinite solutions for the unsteady MHD motion of the fluid in an orthogonal rheometer in which the non-torsional oscillations are superposed on the disks are established. These solutions for $f$ and $g$ reduce to the solutions of Rao and Kasiviswanathan [10] for $B_{0} \rightarrow 0$. 


\section{Results and discussion}

In view of Eqs. (15) and (16) the velocity components are given by

$$
u=-\omega(y-g(z, t)), \quad v=\omega(x-f(z, t)), \quad w=0 .
$$

Then the components $(\xi, \eta, \zeta)$ of the vorticity are given by

$$
\xi=\omega f_{z}, \quad \eta=\omega g_{z}, \quad \zeta=2 \omega
$$

where $f$ and $g$ are given by Eqs. (25) and (26). Substituting Eqs. (14) and (16) in Eqs. (8) and (9), we get

$$
\begin{aligned}
& \phi_{x}=\omega^{2} x+\omega\left(v g_{z z}-\omega f-g_{t}-\frac{\sigma B_{0}^{2}}{\rho} g\right)+\frac{\sigma B_{0}^{2}}{\rho} \omega y, \\
& \phi_{y}=\omega^{2} y-\omega\left(v f_{z z}+\omega g-f_{t}-\frac{\sigma B_{0}^{2}}{\rho} f\right)-\frac{\sigma B_{0}^{2}}{\rho} \omega x .
\end{aligned}
$$

Using Eqs. (11), (25) and (26) in (56) we obtain

$$
\begin{aligned}
& p+\rho \Omega=\frac{\rho \omega^{2}}{2}\left(1-\frac{\sigma^{2} B_{0}^{4}}{\rho^{2} \omega^{2}}\right)\left[\frac{\left(x-X_{0}\right)^{2}}{\left(1+\frac{\sigma B_{0}^{2}}{\rho \omega}\right)}+\frac{\left(y-Y_{0}\right)^{2}}{\left(1-\frac{\sigma B_{0}^{2}}{\rho \omega}\right)}\right]+p_{0}, \quad B_{0}^{2}<\frac{\rho \omega}{\sigma}, \\
& p+\rho \Omega=\frac{\rho \omega^{2}}{2}\left(\frac{\sigma^{2} B_{0}^{4}}{\rho^{2} \omega^{2}}-1\right)\left[\frac{\left(y-Y_{0}\right)^{2}}{\left(\frac{\sigma B_{0}^{2}}{\rho \omega}-1\right)}-\frac{\left(x+X_{0}\right)^{2}}{\left(\frac{\sigma B_{0}^{2}}{\rho \omega}+1\right)}\right]+p_{0}, \quad B_{0}^{2}>\frac{\rho \omega}{\sigma}, \\
& p+\rho \Omega=\rho \omega^{2}\left[\left(x-X_{0}\right)^{2}-4 Y_{0} y\right]+p_{0}, \quad \frac{\sigma^{2} B_{0}^{4}}{\rho^{2} \omega^{2}}=1
\end{aligned}
$$

where $p_{0}$ is an arbitrary constant, $X_{0}(h, t), Y_{0}(h, t)$ are given by

$$
\begin{aligned}
X_{0}= & x_{p}\left(1+\phi_{0}(h) / \Delta_{20}\right)+y_{p} \chi_{0}(h) / \Delta_{20} \\
& -\left[\left(1+\frac{\delta}{\omega}\right)\left\{\left(b_{2} \chi_{3}(h)+b_{1} \phi_{3}(h)\right) / \Delta_{23}-a_{1}^{\prime}\right\}\right. \\
& \left.+\left(1-\frac{\delta}{\omega}\right)\left\{\left(d_{1} \phi_{1}(h)+d_{2} \chi_{1}(h)\right) / \Delta_{21}-b_{1}^{\prime}\right\}\right] \cos \delta t \\
& -\left[\left(1+\frac{\delta}{\omega}\right)\left\{\left(b_{1} \chi_{3}(h)-b_{2} \phi_{3}(h)\right) / \Delta_{23}+a_{2}^{\prime}\right\}\right. \\
& \left.+\left(1-\frac{\delta}{\omega}\right)\left\{\left(d_{2} \phi_{1}(h)-d_{1} \chi_{1}(h)\right) / \Delta_{21}-b_{2}^{\prime}\right\}\right] \sin \delta t \\
& -\frac{N v}{\omega}\left[-y_{p}\left(1-\phi_{0}(h) / \Delta_{20}\right)-x_{p} \chi_{0}(h)\right. \\
& +\left\{\left(b_{2} \phi_{3}(h)-b_{1} \chi_{3}(h)\right) / \Delta_{23}+\left(d_{1} \chi_{1}(h)-d_{2} \phi_{1}(h)\right) / \Delta_{21}-a_{2}^{\prime}-b_{2}^{\prime}\right\} \cos \delta t \\
& \left.+\left\{\left(b_{1} \phi_{3}(h)+b_{2} \chi_{3}(h)\right) / \Delta_{23}-\left(d_{1} \phi_{1}(h)+d_{2} \chi_{1}(h)\right) / \Delta_{21}-a_{1}^{\prime}+b_{1}^{\prime}\right\} \sin \delta t\right], \\
Y_{0}= & y_{p}\left(1+\phi_{0}(h) / \Delta_{20}\right)-x_{p} \chi_{0}(h) / \Delta_{20} \\
+ & {\left[\left(1+\frac{\delta}{\omega}\right)\left\{\left(b_{1} \chi_{3}(h)-b_{2} \phi_{3}(h)\right) / \Delta_{23}+a_{2}^{\prime}\right\}\right.}
\end{aligned}
$$




$$
\begin{aligned}
& \left.+\left(1-\frac{\delta}{\omega}\right)\left\{\left(d_{1} \chi_{1}(h)-d_{2} \phi_{1}(h)\right) / \Delta_{21}+b_{2}^{\prime}\right\}\right] \cos \delta t \\
& +\left[\left(1+\frac{\delta}{\omega}\right)\left\{a_{1}^{\prime}-\left(b_{2} \chi_{3}(h)+b_{1} \phi_{3}(h)\right) / \Delta_{23}\right\}\right. \\
& \left.+\left(1-\frac{\delta}{\omega}\right)\left\{\left(d_{1} \phi_{1}(h)+d_{2} \chi_{1}(h)\right) / \Delta_{21}-b_{1}^{\prime}\right\}\right] \sin \delta t \\
& +\frac{N v}{\omega}\left[-x_{p}\left(1+\phi_{0}(h) / \Delta_{20}\right)-y_{p} \chi_{0}(h) / \Delta_{20}\right. \\
& +\left\{\left(b_{1} \phi_{3}(h)+b_{2} \chi_{3}(h)\right) / \Delta_{23}+\left(d_{1} \phi_{1}(h)+d_{2} \chi_{1}(h)\right) / \Delta_{21}-a_{1}^{\prime}-b_{1}^{\prime}\right\} \cos \delta t \\
& \left.+\left\{\left(d_{2} \phi_{1}(h)-d_{1} \chi_{1}(h)\right) / \Delta_{21}-\left(b_{2} \phi_{3}(h)-b_{1} \chi_{3}(h)\right) / \Delta_{23}+a_{2}^{\prime}-b_{2}^{\prime}\right\} \sin \delta t\right] .
\end{aligned}
$$

The results (57)-(59) reduce to the results of Rao and Kasiviswanathan [10] in the absence of MHD and Berker [4] in the absence of MHD and oscillations.

The components of stress $t_{1}$ applied by the fluid at any point $(x, y, h)$ on the upper disk in $(x, y, z)$ directions are given by

$$
t_{1 x}=-\mu\left(u_{z}+w_{x}\right), \quad t_{1 y}=-\mu\left(v_{z}+w_{y}\right), \quad t_{1 z}=p-2 \mu w_{z},
$$

where $p$ is the pressure and $\mu$ the coefficient of viscosity. Using the values of $(u, v, w)$ given in Eq. (54), we obtain from Eq. (60)

$$
t_{1 x}=-\mu \omega g_{z}(t, h), \quad t_{1 y}=\mu \omega f_{z}(t, h), \quad t_{1 z}=p,
$$

where $f$ and $g$ are defined in Eqs. (48) and (49). Similarly, the stress applied on the lower plate at any point $(x, y,-h)$ can be calculated.

A number of interesting special cases can be derived from the general solutions given by Eq. (45). A solution is said to be symmetric solution with respect to a given point $O$, if the velocity field is a symmetric vector field with respect to the point $O$. Therefore, the symmetric solution has to satisfy the following condition:

$$
\mathbf{V}(x, y, z)=-\mathbf{V}(x, y, z) .
$$

Among the solutions obtained for the motion in the orthogonal rheometer, we need to find whether there exist solutions which are symmetric with respect to: (i) the point $O$, (ii) the mid point of the line segment $P_{1} P_{2}$. The velocity field given by Eq. (54) satisfies the condition (62), provided

$$
f(-z, t)=-f(z, t) \quad \text { and } \quad g(-z, t)=-g(z, t)
$$

which implies that $f$ and $g$ are odd functions of $z$ and

$$
f(0)=0, \quad g(0)=0 .
$$

The latter equation shows that the curve $\Gamma$ must pass through the origin $O$ of the axes. Therefore, for a symmetric solution the constants in Eq. (45) should satisfy

$$
\bar{c}+\bar{a}-2 a_{0}=\bar{d}+\bar{b}-2 b_{0}=0, \quad a_{0}=b_{0}=x_{p}=y_{p}=0 .
$$


Thus, for a symmetric solution all the arbitrary constants given in the boundary condition (44) vanish and the solution thus obtained is unique. This symmetric solution is given by

$$
\begin{aligned}
W= & \left\{\frac{\bar{c}-\bar{a}}{2} \frac{\sinh \sqrt{N+\lambda_{3}^{2}} z}{\sinh \sqrt{N+\lambda_{3}^{2}} h}\right\} e^{i \delta t}+\left\{\frac{\bar{d}-\bar{b}}{2} \frac{\sinh \sqrt{N+\lambda_{1}^{2}} z}{\sinh \sqrt{N+\lambda_{1}^{2}} h}\right\} e^{-i \delta t} \\
& +\left(x_{1}+i y_{1}\right) \frac{\sinh \sqrt{N+\lambda_{0}^{2}} z}{\sinh \sqrt{N+\lambda_{0}} h} .
\end{aligned}
$$

This solution includes the symmetric solution of Kasiviswanathan and Rao [13] as a special case. Taking $y_{1}=0$ and $y_{P}=0$ in Eq. (45), we obtain the solutions of the MHD flow due to non-torsionally oscillating eccentric rotating disks given by Kasiviswanathan and Rao [13]. In the limit $B_{0} \rightarrow 0$ in Eq. (45), we obtain solutions given by Rao and Kasiviswanathan [10]. By taking $B_{0} \rightarrow 0, \delta \rightarrow 0$ (together with the amplitudes of the oscillations) in Eq. (45), we get

$$
W(z)=\left(x_{1}+i y_{1}\right) \frac{\sinh \lambda_{0} z}{\sinh \lambda_{0} h}+\left(x_{P}+i y_{P}\right)\left[1-\frac{\cosh \lambda_{0} z-1}{\cosh \lambda_{0} z-1}\right],
$$

which corresponds to the solutions given by Berker [4]. As $\delta \rightarrow 0, B_{0} \rightarrow 0, \omega \rightarrow 0$ in Eq. (45), we get

$$
\begin{aligned}
W(z)= & {\left[\frac{\bar{c}+\bar{a}-2 a_{0}}{2}+\frac{\bar{d}+\bar{b}-2 b_{0}}{2}-\left(x_{P}+i y_{P}\right)\right] \frac{z^{2}}{h^{2}} } \\
& +\left[\frac{\bar{c}-\bar{a}}{2}+\frac{\bar{d}-\bar{b}}{2}+x_{1}+i y_{1}\right] \frac{z}{h}+\left(x_{P}+i y_{P}+a_{0}+b_{0}\right),
\end{aligned}
$$

and the corresponding boundary conditions are

$$
\begin{aligned}
& W(h)=x_{1}+i y_{1}+\bar{c}+\bar{d}, \quad W(-h)=-\left(x_{1}+i y_{1}\right)+\bar{a}+\bar{b}, \\
& W(0)=x_{P}+i y_{P}+a_{0}+b_{0} .
\end{aligned}
$$

This case corresponds to the flow of a fluid confined between two plates moving with velocities of different magnitudes and direction. It reduces to the solutions given by Kasiviswanathan and Rao [13] for $y_{P}=0$ and $y_{1}=0$. Since in the limit $\omega \rightarrow 0$, all the velocity components given in Eq. (15) become zero; therefore we cannot take this velocity field for the above problem. However, in order to understand the nature and physical significance of this case, we choose the velocity field given by Kasiviswanathan and Rao [13]. We have

$$
u=f(z), \quad v=g(z), \quad w=0 .
$$

In the absence of a magnetic field, Eq. (1) in view of Eq. (70) yields

$$
\mu f_{z z}-p_{x}=0, \quad \mu g_{z z}-p_{y}=0, \quad p_{z}=0 .
$$

Eliminating the pressure gradient we obtain

$$
W_{z z z}=0 \text {. }
$$


The boundary conditions are given by

$$
\begin{aligned}
& W(h)=u_{1}+i u_{1}^{\prime}=U_{1}, \quad W(-h)=u_{2}+i u_{2}^{\prime}=U_{2}, \\
& W(0)=u_{3}+i u_{3}^{\prime}=U_{3},
\end{aligned}
$$

where $U_{3}$ is chosen arbitrarily. The solution of Eq. (72) subject to the boundary conditions (73) yields

$$
W(z)=\frac{1}{2}\left[U_{1}+U_{2}-2 U_{3}\right] \frac{z^{2}}{h^{2}}+\frac{1}{2}\left[U_{1}-U_{2}\right] \frac{z}{h}+U_{3},
$$

which is similar to the solution given in Eq. (68). The solution given by the above equation yields a set of infinite number of solutions as $U_{3}$ is chosen arbitrarily. For $U_{1}=-U_{2}$ and $U_{3}=0$, Eq. (74) reduces to a simple shearing motion and the solution so obtained is a symmetric solution. The stream function for this flow is given by

$$
\psi(z)=y f(z)-x g(z)+C
$$

and the streamlines are given by a family of parallel straight lines in each of the plane where $z=$ constant. In Eq. (75), $C$ denotes an arbitrary constant.

\section{Conclusion}

In this study we have considered the unsteady MHD flow of a viscous fluid in which each point of the parallel planes is subjected to the non-torsional oscillations in their own planes. Exact solutions for the described model are presented. The major findings of the present work are summarised as follows:

- The existence of three sets of infinite numbers of solutions for the unsteady MHD motion of a viscous fluid in an orthogonal rheometer in which the non-torsional oscillations are superposed on the disks is established. These solutions reduce to a single unique solution when symmetric solutions are looked for.

- The results of Rao and Kasiviswanathan [10] are recovered for $B_{0}=0$ and Berker [4] in the absence of MHD and oscillations.

- The present work also includes the solutions of Kasiviswanathan and Rao [13] as a special case.

Competing interests

The authors have no competing interests.

Authors' contributions

All authors contributed equally to the writing of this paper. All authors read and approved the final manuscript.

\section{Author details}

${ }^{1}$ Department of Basic Sciences, Riphah International University, Sector I-14, Islamabad, 44000, Pakistan. ${ }^{2}$ Department of Mathematics, COMSATS Institute of Information Technology, Attock, Pakistan. ${ }^{3}$ Department of Mathematics, COMSATS Institute of Information Technology, Abbottabad, Pakistan.

Received: 15 November 2013 Accepted: 28 May 2014 Published online: 09 August 2014

\section{References}

1. Berker, RA: Sur Quelques cas d' intéegration des équations du mouvement d'un fluide visqueux incompressible. Théses françaises de l'entre-deux-guerres (1936)

2. Berker, R: Intégration des équations du mouvement d'un fluide visqueux incompressible. In: Handbuch der Physik, vol. VIII/2, pp. 1-384. Springer, Berlin (1963) 
3. Berker, R: A new solution of the Navier-Stokes equation for the motion of a fluid contained between two parallel plates rotating about the same axis. Arch. Mech. Stosow. 31, 265-280 (1979)

4. Berker, R: An exact solution of the Navier-Stokes equation. The vortex curvilinear axis. Int. J. Eng. Sci. 20, 217-230 (1982)

5. Rajagopal, KR, Gupta, AS: Flow and stability of second grade fluid between rotating planes. Arch. Mech. 33, 663-674 (1981)

6. Rajagopal, KR, Gupta, AS: Flow and stability of a second grade fluid between two parallel plates rotating about noncoincident axes. Int. J. Eng. Sci. 19, 1401-1409 (1981)

7. Rajagopal, KR: On the flow of a simple fluid in an orthogonal rheometer. Arch. Ration. Mech. Anal. 79, 39-47 (1982)

8. Siddiqui, AM, Rana, MA, Ahmed, N: Effects of Hall current and heat transfer on MHD flow of a Burgers' fluid due to a pull of eccentric rotating disks. Commun. Nonlinear Sci. Numer. Simul. 13, 1554-1570 (2008)

9. Siddiqui, AM, Rana, MA, Ahmed, N: Magnetohydrodynamics flow of a Burgers' fluid in an orthogonal rheometer. Appl. Math. Model. 34, 2881-2892 (2010)

10. Rao, AR, Kasiviswanathan, SR: On exact solutions of the unsteady Navier-Stokes equations-the vortex with instantaneous curvilinear axis. Int. J. Eng. Sci. 25, 337-349 (1987)

11. Abbott, TNG, Walters, K: Rheometrical flow systems, part 2. Theory for the orthogonal rheometer, including an exact solution of the Navier-Stokes equations. J. Fluid Mech. 40, 205-213 (1970)

12. Thornley, C: On Stokes and Rayleigh layers in a rotating system. Q. J. Mech. Appl. Math. 21, 451-461 (1968)

13. Kasiviswanathan, SR, Rao, AR: On exact solutions of unsteady MHD flow between eccentrically rotating disks. Arch. Mech. 39, 411-418 (1987)

14. Mohanty, HK: Hydromagnetic flow between two rotating disks with noncoincident parallel axes of rotation. Phys. Fluids 15, 1456-1458 (1972)

15. Erkman, CS: Magnetohydrodynamic flow of a viscous, incompressible fluid between rotating parallel plates. Lett. Appl. Eng. Sci. 3, 51-59 (1975)

16. Murthy, SN, Ram, RKP: MHD flow and heat transfer due to eccentric rotations of a porous disk and a fluid at infinity. Int. J. Eng. Sci. 16, 943-949 (1978)

17. Rao, AR, Rao, PR: On the magnetohydrodynamic flow between eccentrically rotating disks. Int. J. Eng. Sci. 21, 359-372 (1983)

18. Ersoy, HV: MHD flow of an Oldroyd-B fluid between eccentric rotating disks. Int. J. Eng. Sci. 37, 1973-1984 (1999)

19. Rajagopal, KR: Flow of viscoelastic fluids between rotating plates. Theor. Comput. Fluid Dyn. 3, 185-206 (1992)

20. Sherchiff, J: A Textbook of Magnetohydrodynamics. Pergamon, Oxford (1965)

21. Davidson, PA: An Introduction to Magnetohydrodynamics, 1st edn. Cambridge University Press, Cambridge (2001)

22. Sutton, GW, Sherman, A: Engineering Magnetohydrodynamics. McGraw-Hill, New York (1965)

doi:10.1186/s13661-014-0146-y

Cite this article as: Rana et al.: On exact solution of unsteady MHD flow of a viscous fluid in an orthogona

rheometer. Boundary Value Problems 2014 2014:146.

\section{Submit your manuscript to a SpringerOpen ${ }^{\ominus}$ journal and benefit from:}

- Convenient online submission

- Rigorous peer review

- Immediate publication on acceptance

Open access: articles freely available online

- High visibility within the field

- Retaining the copyright to your article 